\title{
Membership and Fees / Admission et cotisation
}

Membership in either the Canadian Political Science Association or the Société québécoise de science politique includes a subscription to the Canadian Journal of Political Science. There are two levels of membership fees. Income level stated refers to gross annual income (before taxes). Members may subscribe to The Canadian Review of Sociology and Anthropology and/or Canadian Public Policy at a special rate.

La cotisation à l'Association canadienne de science politique ou à la Société québécoise de science politique comprend un abonnement à la Revue canadienne de science politique. Il y a deux niveaux de frais de cotisation dans chaque catégorie. Le montant de la cotisation est déterminé en fonction de votre revenu annuel brut (avant impôt). En tant que membre il vous est possible de vous abonner à un prix avantageux à la Revue canadienne de Sociologie et d'Anthropologie et/ou L'Analyse de politiques.

\begin{tabular}{lcc} 
& $\begin{array}{l}\text { Students, retired or unemployed } \\
\text { individuals and any other person } \\
\text { earning under } \$ 15,000 \text { annually/ } \\
\text { Étudiant(e)s, personnes retraites } \\
\text { ou sans travail et toutes autres } \\
\text { personnes dont le revenu annuel } \\
\text { ne dépasse pas 15000\$ }\end{array}$ & $\begin{array}{l}\text { All } \\
\text { other } \\
\text { members/ }\end{array}$ \\
$\begin{array}{l}\text { The choice of Associations/ } \\
\text { Choix d'associations }\end{array}$ & $\begin{array}{l}\text { autres } \\
\text { membres }\end{array}$ \\
\hline $\begin{array}{l}\text { CPSA only/ACSP seulement } \\
\text { SQSP seulement/only* }\end{array}$ & $\$ 30.00$ & $\$ 80.00$ \\
$\begin{array}{l}\text { Joint/conjoint CPSA/ACSP-SQSP* } \\
\text { *These categories of membership include } \\
\text { a subscription to the SQSP's journal } \\
\text { Politique. / Ces catégories d'adhésion } \\
\text { comprennent un abonnement à la revue } \\
\text { Politique de la SQSP. }\end{array}$ & $\$ 30.00$ & $\$ 80.00$ \\
\hline
\end{tabular}

Institutional subscription- $\$ 60.00$ in Canada; $\$ 80.00$ in member countries of OECD; $\$ 60.00$ in all other countries

Abonnement institutionnel- $60 \$$ au Canada; $80 \$$ dans les pays-membres de l'OCDE; $60 \$$ dans tout autre pays

For information, please write:/

Pour des renseignements, veuillez vous adresser à :

The Canadian Journal of Political Science/

La Revue canadienne de science politique

12 Henderson Avenue

Université d'Ottawa

Ottawa, Ontario K1N 6N5 